Meta

Journal des tradlucteurs

Translators' Journal

\title{
Une révolution qui n'en a pas l'air
}

\section{Robert Dubuc}

Volume 21, numéro 1, mars 1976

La traduction et l'entreprise

URI : https://id.erudit.org/iderudit/002629ar

DOI : https://doi.org/10.7202/002629ar

Aller au sommaire du numéro

Éditeur(s)

Les Presses de l'Université de Montréal

ISSN

0026-0452 (imprimé)

1492-1421 (numérique)

Découvrir la revue

Citer cet article

Dubuc, R. (1976). Une révolution qui n'en a pas l'air. Meta, 21(1), 16-21.

https://doi.org/10.7202/002629ar d'utilisation que vous pouvez consulter en ligne.

https://apropos.erudit.org/fr/usagers/politique-dutilisation/ 


\section{Une révolution qui n'en a pas l'air}

L'histoire du français langue de travail au Québec a été longtemps celle đu voyage du pot de terre et du pot de fer. À côté du pot de fer anglais, tout-puissant, apanage des patrons, clé des promotions, le pot de terre français s'estimait heureux lorsqu'il pouvait de peine et de misère se maintenir à flot. Mais le plus souvent, il volait en éclat sous le choc de ce voisinage brutal et laissait ses débris emportés dans le courant d'une prospérité achetée au prix de son intégrité.

\section{Une évolution révolutionnaire}

Mais voici qu'un élément nouveau intervient. À la suite d'une prise de conscience collective, le législateur entend introduire, par la grande porte, le français dans la vie de l'entreprise. Il n'en fallait pas moins pour produire une véritable révolution.

Cette donnée risque en effet de transformer ce qu'on pourrait appeler l'« écologie » linguistique de l'entreprise québécoise. Cette révolution va faire sentir son action sur plusieurs points.

\section{Respect de la personnalité du francophone}

Le premier touché, c'est évidemment le francophone lui-même à l'intérieur de l'entreprise. La reconnaissance officielle de sa langue le dispensera désormais de la mutation de mentalité, de cette sorte de travestissement de son être profond auquel l'obligeait l'adoption d'une langue seconde comme langue première. Il pourra dorénavant gagner sa vie dans sa propre langue, c'est-à-dire en restant profondément lui-même. La langue en effet touche aux fibres mêmes de l'être.

Si la langue que nous parlons n'est utile qu'à un niveau domestique, nous développons par le fait même une mentalité de domestique. Si, par contre, cette langue peut permettre la promotion sociale de l'individu, elle contribue à son enrichissement. C'est pourquoi, même si cette idée s'est quelque peu déflorée en devenant un slogan politique, le français, langue de travail, devrait être le premier élément, la pierre d'angle de toute politique linguistique. Une langue dont on ne se sert pas pour gagner son pain devient vite un luxe qu'on n'a pas toujours les moyens de s'offrir. En faisant du français une langue de communication dans le milieu du travail, l'État évite au francophone d'être infériorisé par sa langue. 


\section{Véritable place de la traduction}

Le second front de cette révolution, c'est la traduction. Les entreprises font, à l'heure actuelle, des traductoses aiguës. Sous l'aiguillon de la Loi 22, on découvre soudain une réalité où le français a été totalement absent - ou presque. On veut alors faire du rattrapage par le biais de la traduction. On veut tout traduire pour au moins se donner bonne conscience et obtenir de l'État son brevet de bonne conduite.

Toutefois, croire qu'on a instauré le français comme langue de travail parce qu'on traduit tout c'est un leurre. La politique du «tout-traduit » peut fort bien n'être qu'un paravent pour pallier l'absence des francophones aux postes de commande dans des fonctions de conception et de création. Par le passé, si d'aventure dans une entreprise anglophone un Canadien français ingénieur, chimiste ou comptable parvenait à se hisser au niveau des cadres, ne voyait-il pas souvent ses fonctions ramenées à celles d'un traducteur? On changeait l'étiquette sur la bouteille, mais le contenu était le même.

Si l'on ne se gargarise pas de mots, le français, langue de travail, doit de toute nécessité permettre aux francophones de créer et de produire dans leur langue. Alors la traduction reprendra son rôle normal d'intermédiaire de communication entre deux groupes linguistiques distincts et non plus celui d'agent général de communication entre une haute direction essentiellement anglophone et la piétaille et la valetaille francophones. C'est en respectant la nature des choses que celles-ci nous servent le mieux.

\section{Enrichissement et épuration du français}

La troisième cible de cette révolution sera la langue française elle-même, qu'il faudra enrichir et épurer pour la mettre au diapason des besoins.

Il est difficile de passer de l'état de langue artisanale à celui de véhicule de communication dans une société hautement technique. Cela suppose une métamorphose dont le moins qu'on puisse dire c'est qu'elle comporte des moments pénibles.

Le français québécois a été depuis 1763 jusqu'à une date très récente une simple langue d'expression pour les besoins vitaux essentiels, la communication s'établissant le plus souvent à ce niveau. C'est d'ailleurs ce qui explique pour une bonne part la force émotive véhiculée par la littérature populaire d'ici dont Deschamps et Tremblay fournissent d'excellents exemples.

Cette langue, au niveau de l'expression personnelle, a certes droit de cité. On ne peut contester à nos littérateurs issus d'un peuble dont la souffrance et l'humiliation ont été le pain quotidien, le droit de proférer ce cri du ventre dans un effort pour exorciser ses démons intérieurs. La brutalité et le caractère fruste de ce langage lui confère un impact émotif dont on ne peut ignorer l'intensité. Il y a de la vérité là-dedans. Toute vérité appelle le respect.

On voit difficilement toutefois comment cette langue pourrait servir à un rapport administratif ou à établir une description de tâche. C'est qu'au niveau de la langue de travail, la langue n'est plus fondamentalement le véhicule d'un lyrisme personnel ou collectif, mais bien d'abord et avant tout un moyen de communication. 
Communiquer à ce niveau, ce n'est plus faire entendre un cri, c'est pouvoir rendre avec précision toutes les nuances de sa pensée, donner forme dans un discours clair et bien charpenté aux éléments constitutifs de ses conceptions et de ses créations. Pour y parvenir il faut disposer d'une langue nuancée, structurée, capable tout autant d'images que d'abstraction. Cette langue pour nous, c'est le français. Certains démagogues cherchent à nous convaincre que le français est pour nous une langue étrangère. Mais on oublie alors qu'historiquement nous tenons à la langue française par toutes nos fibres. Notre folklore est de pure souche française. Notre résistance à l'assimilation s'est toujours greffée sur l'amour de cette langue considérée comme un héritage sacré. Toute notre histoire, comme peuple, est jalonnée de témoignages de fidélité à la langue française. Toute la tradition de notre enseignement et de nos échelles de valeurs a été également imprégnée de culture française. La langue française, c'est notre bien à nous. Qu'on ne cherche pas, par démagogie, à nous en spolier. Cet héritage, il nous appartient maintenant de le faire fructifier, de l'enrichir et de l'épurer.

\section{Le français, langue difficile?}

Certains mandarins du langage se sont plu à présenter le français sous son jour le plus ardu. Je veux bien m'émouvoir devant les scrupules d'une Colette lorsqu'elle écrit : "C'est une langue bien difficile que le français. À peine écrit-on depuis quarante-cinq ans qu'on commence à s'en apercevoir. » On n'est pas tous tenu d'écrire comme Colette. J'aurais plutôt tendance à croire qu'il n'est aucun domaine où le mieux soit davantage l'ennemi du bien que celui de la langue.

Le français, langue difficile? Toute langue évoluée a ses aspérités. Écrire et parler correctement, honnêtement le français n'est au-dessus des forces d'aucune personne capable de gagner sa vie. L'essence de la langue française ne tient pas à la règle de l'accord de ci-joint, ci-inclus. Il tient à une structure syntaxique simple où les rapports entre les mots sont transparents. Pour maitriser la syntaxe du français, il faut et il suffit de connaître sa grammaire. Ne demande-t-on pas au médecin de connaître l'anatomie, au chimiste, la chimie? N'est-il pas normal d'exiger de celui dont la langue est le gagne-pain qu'il sache sa grammaire ? L'école, hélas ! n'y croit plus. Il nous reste donc à l'apprendre par nous-mêmes. Il suffirait d'une heure d'étude hebdomadaire pendant six mois pour y parvenir. C'est un investissement qui en vaut d'autres et surtout... c'est possible. Quelle grammaire choisir? Les bonnes vieilles grammaires classiques sont encore la meilleure voie pour acquérir une connaissance systématique de la langue.

Il faut dire que la correction de la langue n'est pas une fin en soi. C'est une manière de communiquer efficacement. $\grave{A}$ ce propos, il n'est pas sans intérêt de rappeler les conclusions d'une enquête menée par Sorecom, il y a quelques années, sur la publicité. On avait proposé à un échantillon représentatif de la population en général les textes d'une vingtaine d'annonces publicitaires de qualités diverses et de niveaux de langue divers. Or, dans la très grande majorité. des cas, ce sont les annonces rédigées en français «normal » qui ont été le mieux comprises.

On voit donc que la grande peur de n'être pas compris, est invoquée à tort contre l'implantation d'une langue française correcte. C'est en écrivant et en parlant 
correctement qu'on est le mieux compris. Ce n'est pas toujours très percutant, mais c'est en général efficace. Le message passe. C'est là l'essentiel.

\section{Obstacles à l'implantation d'une langue de travail forte et vivante}

La situation de la langue technique au Canada pose toutefois des problèmes particuliers pour le vocabulaire, par le fait même que la terminologie technique française est largement inconnue des usagers. Le irançais ayant été à toutes fins pratiques exclu de la langue de travail, il en est résulté une pauvreté du vocabulaire technique qui a ses conséquences pour l'implantation du français dans l'entreprise.

Un lieu commun fort répandu dans les milieux de travail québécois consiste à répéter que le français est une langue inadaptée aux exigences techniques en Amérique du Nord. Cette impression est liée à notre méconnaissance du français technique. Cependant des expériences comme celles de l'Hydro-Québec à la Manic et du métro de Montréal prouvent que ce préjugé est sans fondement dans la réalité. Le français possède toutes les ressources nécessaires pour exprimer les réalités techniques, au moins virtuellement. Les obstacles à son utilisation sont beaucoup plus psychologiques que linguistiques.

Le premier obstacle est relié à l'apprentissage des termes. Notre éloignement du français de la métropole, notre voisinage de l'américain ont fait que nous n'avons pas toujours appris les «bons » termes techniques. Or, le point où l'esprit, pour la première fois, associe une notion avec un mot ou une expression est un moment privilégié - qu'il s'agisse d'un appareil ou d'un concept philosophique, le réflexe est le même. Il se crée alors entre la notion et son appellation une sorte de lien essentiel qui rend ensuite très difficile toute opération de substitution.

Nous réagissons tous un peu négativement face à un terme technique français inconnu. Un taxiphone? ça ne peut être un téléphone payant! Un enjoliveur, c'est bien trop compliqué quand on peut dire un «cap de roue »! Cette mentalité pose des obstacles sérieux à l'implantation d'un français ferme et vigoureux dans le milieu du travail. Tant qu'on s'acharnera à prendre pour norme chacun son «petit français », il ne sera pas possible d'avoir une langue de communication efficace dans l'entreprise. Elle sera trop étroite.

En somme, il faudrait pratiquer une certaine ouverture d'esprit à l'égard des mots nouveaux et des acquisitions qui nous obligent à modifier nos habitudes de langage. Autrement, on se leurre. J'ai lu un jour dans un rapport technique d'entretien: «J'ai resserré la noix qui tient les mains de l'horloge. » Est-ce vraiment cela, le français langue de travail ?

Quoi qu'en pensent certains néo-linguistes, mal frottés de marxisme, la langue d'atelier n'est pas nécessairement promue au rang de langue de culture par le simple fait d'exister ${ }^{1}$.

1. Voir à ce propos la théorie d'Alphonso Caramazza, citée par Joel McCormick, dans Extra 1, Concordia University, vol. 1, $\mathrm{n}^{\circ} 1$, avril 1975, p. 14: "Culture includes the language spoken on the shop floor and the government, through industrial take-overs if necessary, should do everything in its power to preserve it. " 
Une langue de travail, distincte de l'anglais, n'a de chance de subsister au Québec que si elle est fondamentalement française, c'est-à-dire si elle peut trouver appui dans une communauté linguistique élargie. Le sort d'une langue québécoise autonome est voué dès le départ à une vie végétative, comme le breton en France et le gallois en Grande-Bretagne.

Dans son état actuel, en bien des milieux, la langue de travail au Québec n'est que le baromètre de notre anglicisation. C'est dire que l'implantation du français comme langue de travail implique un sérieux effort de recherche et de diffusion de la terminologie. Cet effort est déjà amorcé par la Régie de la langue française du Québec, par la Banque de terminologie de l'Université de Montréal et par le Centre de terminologie du Secrétariat d'État à Ottawa. Mais ces efforts, par leur dispersion même, portent en eux un germe de mort. Depuis cinq ans, il n'a pas été possible de conjuguer ces efforts dispersés, faute d'une conscience claire des problèmes et des solutions qu'ils réclament, faute aussi d'une largeur de vue suffisante et nécessaire à toute collaboration. Les gouvernements ont fait preuve de part et d'autre d'un chauvinisme aussi stérile que dépourvu de réalisme. Pendant qu'on se chamaillait pour s'assurer une hégémonie illusoire, on laissait pourrir le problème, apparemment indifférent aux exigences de l'efficacité, du rendement et de la qualité. Ce n'est certes pas ainsi que nous ferons progresser le français, langue de travail.

Il ne faut pas oublier en effet que dans cette conjoncture il y a un facteur qui domine souvent tous les autres : c'est l'omniprésence de la langue anglaise.

Tout favorise cette dernière: hégémonie économique et technique, grand nombre d'utilisateurs, position de force dans tous les secteurs de l'activité industrielle. Il va de soi qu'une telle puissance ne manque pas d'exercer une très grande influence, surtout pour nous qui sommes, pour ainsi dire, sur la ligne de feu. Cette influence se trouve encore compliquée du fait de la parenté des langues en présence. Beaucoup de mots français et anglais ont des formes analogues, mais des sens différents. Comment savoir qu'en employant en anglais le mot «versatile», on fait un compliment à quelqu'un, tandis que le mot français versatile s'applique à un défaut: l'instabilité. La forme est identique, mais le sens différent. C'est une difficulté sérieuse, mais encore ici le seul moyen de s'en sortir, c'est de savoir. Heureusement, les bons outils ne manquent plus. Radio-Canada a mis au point un fichier où sont traités près de 2000 anglicismes ${ }^{2}$, M. Gilles Colpron a fait une étude approfondie des anglicismes au Québec ${ }^{3}$ et $\mathbf{M}$. Gérard Dagenais a publié un dictionnaire des Difficultés de la langue française au Canada ${ }^{4}$ où les anglicismes ont la part du lion. J'ai moi-même réuni dans un recueil les 200 fautes les plus courantes de notre parler avec des petits exercices correctifs ${ }^{5}$. Il reste à savoir. Une fois qu'on sait, il n'y a plus de problème.

Le combat contre l'anglicisme se complique encore par l'étendue de la francophonie et la diversité des zones d'influence de la langue anglaise.

2. Service de linguistique, C'est-à-dire et les fiches, Montréal, Société Radio-Canada, depuis 1960 (publication périodique).

3. Gilles Colpron, les Anglicismes au Québec, Montréal, Beauchemin, 1970, 247 p.

4. Gérard Dagenais, Dictionnaire des difficultés de la langue française au Canada, Québec, Pédagogia, $1967,680 \mathrm{p}$.

5. Robert Dubuc, Objectif 200, Montréal, Leméac-Radio-Canada, 1971, 133 p. 
En France, la langue courante connait les anglicismes de snobisme, qui nous choquent tant : footing, parking, dancing, etc. Ce sont les plus insignifiants.

Il y a aussi les anglicismes d'ignorance qui commencent à se répandre. En fait, les Français sont comme nous et réagissent de la même manière au même conditionnement. Les ouvrages techniques et scientifiques, nourris de textes américains, regorgent d'anglicismes et de calques. Quelle attitude devons-nous alors adopter?

\section{Prudence ouverte}

La meilleure attitude à tenir dans ce cas, c'est celle de la prudence. Non pas une prudence hargneuse et stérile, mais une prudence souple et ouverte, capable de s'adapter à une langue en constante évolution. Il faut certes être sur ses gardes face à l'anglicisme, parce que nous y sommes tellement exposés que le moindre relâchement risque de nous entraîner comme une avalanche. C'est cette prudence qui nous rend réticents face à une expression comme promotion des ventes. Mais c'est une ouverture d'esprit nécessaire qui nous incitera à l'accueillir quand elle aura reçu la sanction quasi unanime de l'usage.

Cette souplesse est au fond commandée par la nature profonde d'une langue. Une langue n'obéit pas à des modèles prétablis et parfaits ou à une sorte de "génie » mystérieux qui la guide infailliblement vers la perfection. La réalité est beaucoup plus modeste : la langue, comme le disait si bien Jean-Marie Laurence, n'est qu'un réseau d'habitudes commun à un groupe d'usagers qui acceptent les mêmes conventions. Il nous est possible d'influer partiellement sur ces habitudes et sur ces conventions, mais le plus souvent elles obéissent à des lois obscures sur lesquelles nous n'avons pas grand pouvoir. La sagesse nous incite alors à nous rallier à la loi de la majorité.

\section{Conclusion}

L'évolution de la vie de l'entreprise, par suite de ces transformations, place donc le «Bureau de traduction » classique sur de nouvelles voies. Celui-ci en conséquence se doit d'acquérir suffisamment de souplesse pour s'adapter aux conditions nouvelles. Il s'agira pour lui d'être moins ce corridor de la communication à sens unique qu'un lieu de rencontre des activités linguistiques de l'entreprise : traduction anglais-français et français-anglais, rédaction, communication.

Pour relever ce défi, les traducteurs devront consentir à enlever leurs œillères, qui les ont jusqu'ici confinés à un exercice trop étroit de leur métier. Le traducteur d'aujourd'hui doit aussi être rédacteur, conseiller en rédaction, expert en grammaire et frotté de terminologie pour répondre aux besoins de l'entreprise.

Dans le domaine de la terminologie en particulier, l'effort des entreprises doit reposer sur les bureaux de traduction. Eux seuls semblent vraiment en mesure de concilier les exigences d'une communication efficace dans l'immédiat avec les normes de qualité qu'il faut nécessairement respecter dans une optique d'efficacité à long terme.

Robert Dubuc 